\section{New Theory}

ISSN: 2149-1402
36 (2021) 75-87

Journal of New Theory

https://dergipark.org.tr/en/pub/jnt

Open Access

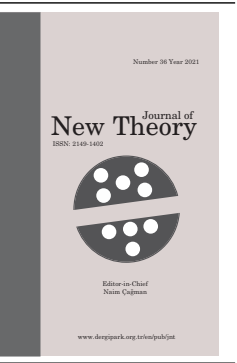

\title{
Construction of Developable Surface with Geodesic or Line of Curvature Coordinates
}

\author{
Nabil Mohammed Althibany ${ }^{1}$ (D)
}

\author{
Article History \\ Received: 26 Aug 2021 \\ Accepted: 20 Sep 2021 \\ Published: 30 Sep 2021 \\ $10.53570 /$ jnt. 987265 \\ Research Article
}

\begin{abstract}
In this paper, a developable surface with geodesic or line of curvature coordinates is constructed in the Euclidean 3-space. A developable surface is coordinated by two families of parametric curves, base curves (directrices) and lines (rulings). Since any part of a straight line on a developable surface is geodesic and line of curvature, we only need to show that the directrices curves are geodesics or lines of curvature to ensure that the developable surface is parameterized by geodesic or line of curvature coordinates. The necessary and sufficient conditions for the directrices curves to be geodesics or lines of curvature are studied. The main results of this paper show that the developable surface with geodesic coordinates is a generalized cylinder, and the developable surface with line of curvature coordinates is a tangent surface.
\end{abstract}

Keywords - Developable surface, geodesic, line of curvature, parametric curves, coordinates

Mathematics Subject Classification (2020) - 53A04, 53A05

\section{Introduction}

A ruled surface is constructed by the continuous motion of a straight line called the ruling or generator through a given curve called the base or directrix curve. Developable surfaces are a special class of the ruled surfaces that can be mapped isometrically into the plane, therefore the developable surface has zero Gaussian curvature as the plane. Cylinders (including planes), cones, and tangent surfaces are three basic types of developable surface. The developable surface has been used in geometric modeling, architecture, and many applications in manufacturing industries [1-3].

Geodesic is a curve that travel in directions of zero geodesic curvature on a surface. The shortest path between two given points on a curved space is given by geodesic. Any (part of ) a straight line on a surface is a geodesic as are the rulings of any ruled surface. Line of curvature is a curve that travel in directions for which the surface curvature takes its maximum or minimum values, in other words, the directions in which a shape bends extremely. The lines of curvatures exists as orthogonal curves on non-umbilical points of the surface. Rulings of any developable surface are lines of curvature.

A coordinate on a surface is said to be geodesic or line of curvature if the two families of coordinate curves are geodesics or lines of curvature. Extracting the geometric information from the surface depends on the parametrization that is using as a coordinate system on the surface. Using geodesic or line of curvature coordinates are suitable for many tasks not only in differential geometry but involves other areas of applications, such as integrable systems [4], surfaces motions [5,6], and architectural shape design $[7,8]$.

\footnotetext{
${ }^{1}$ althibany1972@yahoo.com

${ }^{1}$ Department of Mathematics, Faculty of Applied Science, Taiz University, Taiz, Yemen
} 
In this paper, we discuss how a developable surface can be parameterized by geodesic or line of curvature coordinates. For our purpose, we parameterize the ruled surface by its directrix curve and director vector that is expressed by a linear combination of Frenet frame with angular functions as coefficients. The two families of parametric curves are rulings and directrices. It is well known that the rulings are geodesics and lines of curvature on a developable surface. Consequently, throughout this paper, our focus lies on the family of directrices. Many constraints are assumed on the angular functions through many steps to generate parametrization whose directrices are geodesics or lines of curvature. The main results show that the developable surface with geodesic coordinates is a generalized cylinder, and the developable surface with line of curvature coordinates is a tangent surface.

There are many articles for constructing a developable surface family that possesses the directrix curve as a common characteristic curve, see for example [9-11]. For our approach, [11] is a relevant paper, but it is restricted and limited. More explicitly, it focused on the sufficient and necessary conditions for only one directrix curve to be a line of curvature, whereas our paper deals with conditions on the family of directrices to be geodesics or lines of curvature in the constructed developable surface.

The rest of this paper is organized as follows: In section 2, some basic notations, facts and definitions of the space curves and ruled surfaces are reviewed. The main results are studied in section 3 , where the developable surface with geodesic or line of curvature coordinates is constructed, the necessary and sufficient conditions for the directrices curves to be geodesics or lines of curvature are derived, some geometric properties of such coordinates are analyzed. Examples is presented in section 4. Finally, the conclusion is given in section 5 .

\section{Preliminary}

This section introduces some basic concepts on the classical differential geometry of space curves and ruled surfaces in Euclidean 3-space, as well as some basic definitions and notions that are required subsequently. More details can be found in such standard references as $[12,13]$.

\subsection{Curves in Euclidean 3-space}

A smooth space curve in Euclidean 3-space is parameterized by a map $\gamma: I \subseteq \mathbb{R} \rightarrow E^{3}$, $\gamma$ is called a regular curve if $\gamma^{\prime} \neq 0$ for every point of an interval $I \subseteq \mathbb{R}$, and if $\left|\gamma^{\prime}(s)\right|=1$ where $\left|\gamma^{\prime}(s)\right|=\sqrt{\left\langle\gamma^{\prime}(s), \gamma^{\prime}(s)\right\rangle}$, then $\gamma$ is said to be of unit speed (or parameterized by arc-length $s$ ). For a unit speed regular curve $\gamma(s)$ in $E^{3}$, the unit tangent vector $t(s)$ of $\gamma$ at $\gamma(s)$ is given by $t(s)=\gamma^{\prime}(s)$. If $\gamma^{\prime \prime}(s) \neq 0$, the unit principal normal vector $\mathrm{n}(\mathrm{s})$ of the curve at $\gamma(s)$ is given by $n(s)=\frac{\gamma^{\prime \prime}(s)}{\left\|\gamma^{\prime \prime}\right\|}$. The unit vector $b(s)=t(s) \times n(s)$ is called the unit binormal vector of $\gamma$ at $\gamma(s)$. Physically, the vectors $\gamma^{\prime}(s)$ and $\gamma^{\prime \prime}(s)$ are called the velocity and acceleration vectors respectively. For each point of $\gamma(s)$ where $\gamma^{\prime \prime}(s) \neq 0$, we associate the Serret-Frenet frame $\{t, n, b\}$ along the curve $\gamma$. As the parameter $\mathrm{s}$ traces out the curve, the Serret-Frenet frame moves along $\gamma$ and satisfies the following formula.

$$
\left\{\begin{array}{l}
t^{\prime}(s)=\kappa(s) n(s) \\
n^{\prime}(s)=-\kappa(s) t(s)+\tau b(s) \\
b^{\prime}(s)=-\tau(s) n(s)
\end{array}\right.
$$

where $\kappa=\kappa(s)$ and $\tau=\tau(s)$ are the curvature and torsion functions. The planes spanned by $\{t(s), n(s)\},\{t(s), b(s)\}$ and $\{n(s), b(s)\}$ are respectively called the osculating plane, the rectifying plane and the normal plane. Kinematically, when the point moves along the unit speed curve with non vanishing curvature and torision, the Serret-Frenet frame $\{t, n, b\}$ is drawn to the curve at each position of the moving point, this motion consists of translation with rotation and described by the following Darboux vector

$$
\omega=\tau t+\kappa b
$$

The direction of Darboux vector is the direction of rotational axis and its magnitude gives the 
angular velocity of rotation. The unit Darboux vector field is defined by

$$
\hat{\omega}=\frac{\tau}{\sqrt{\tau^{2}+\kappa^{2}}} t+\frac{\kappa}{\sqrt{\tau^{2}+\kappa^{2}}} b
$$

A necessary and sufficient condition that a curve be of constant slope (or general helix) is that the ratio of curvature to torsion is constant $\left(\frac{\tau}{\kappa}=c\right)$. The general helix lies on a general cylinder and also known as a cylindrical helix. The circular helix ( a helix on a circular cylinder) is a special helix with both of $\kappa(s) \neq 0$ and $\tau(s)$ are constants. The Darboux vector is constant for circular helix. For the cylindrical helix, the unit Darboux vector is constant, where (3) can be written as

$$
\hat{\omega}=\frac{\tau}{\sqrt{\tau^{2}+\kappa^{2}}} t+\frac{\kappa}{\sqrt{\tau^{2}+\kappa^{2}}} b=\frac{\tau / \kappa}{\sqrt{(\tau / \kappa)^{2}+1}} t+\frac{1}{\sqrt{(\tau / \kappa)^{2}+1}} b=\frac{c}{\sqrt{c^{2}+1}} t+\frac{1}{\sqrt{c^{2}+1}} b
$$

For a regular curve on a surface, there exists another frame which is called Darboux frame and denoted by $\{t(s), g(s), N(s)\}$. In this frame $t(s)$ is the unit tangent of the curve, $N(s)$ is the unit normal of the surface and $g$ is a unit vector given by $g=N \times t$. Derivative of the Darboux frame according to arc-length parameter is governed by the following relations

$$
\left\{\begin{array}{l}
t^{\prime}(s)=\kappa_{g} g(s)+\kappa_{n} N(s) \\
g^{\prime}(s)=-\kappa_{g} t(s)+\tau_{g} N(s) \\
N^{\prime}(s)=-\kappa_{n} t(s)-\tau_{g} g(s)
\end{array}\right.
$$

where $\kappa_{g}$ is the geodesic curvature, $\kappa_{n}$ is the normal curvature and $\tau_{g}$ is the geodesic torsion at each point of the curve $\gamma(s)$ which are given by the following

$$
\kappa_{g}=<\gamma^{\prime \prime}(s), g>, \quad \kappa_{n}=<\gamma^{\prime \prime}(s), N>, \quad \text { and } \quad \tau_{g}=<N^{\prime}, g>
$$

The unit tangent $t$ is common in both Frenet frame and Darboux frame, then the vectors $N, g, n$ and $b$ lie on the same plane and the relations between these frames can be given as follows:

$$
\left(\begin{array}{c}
t \\
g \\
N
\end{array}\right)=\left(\begin{array}{ccc}
1 & 0 & 0 \\
0 & \cos \phi & \sin \phi \\
0 & -\sin \phi & \cos \phi
\end{array}\right)\left(\begin{array}{l}
t \\
n \\
b
\end{array}\right)
$$

where

$$
\left\{\begin{array}{l}
g(s)=\cos \phi(s) n(s)+\sin \phi(s) b(s) \\
N(s)=-\sin \phi(s) n(s)+\cos \phi(s) b(s)
\end{array}\right.
$$

Differentiating (8), by using (5) and (1) we get the relation between geodesic curvature, normal curvature, geodesic torsion with curvature, torsion as follows:

$$
\kappa_{g}=\kappa \cos \phi, \quad \kappa_{n}=\kappa \sin \phi, \quad \text { and } \quad \tau_{g}=\tau+\frac{d \phi}{d s}
$$

A unit-speed curve on a surface is a geodesic if and only if its geodesic curvature is zero everywhere $\left(\kappa_{g}=0\right)$ [13]. From (9) and (8), this condition is equivalent to the following

$$
N= \pm n
$$

Hence a curve is a geodesic on the surface if and only if the principal normal $n$ to the curve and the surface normal $N$ are parallel to each other at any point on the curve. Equivalently, a curve $\gamma(s)$ on the surface is a geodesic provided its acceleration vector $\gamma^{\prime \prime}(s)$ is always normal to the surface, i.e.

$$
\gamma^{\prime \prime}(s) \times N=0
$$

A unit-speed curve on a surface is a line of curvature if and only if its geodesic torsion is zero everywhere $\left(\tau_{g}=0\right)$ [13]. From (9), this condition is equivalent to the following

$$
\tau+\frac{d \phi}{d s}=0
$$




\subsection{Ruled Surfaces}

Let $X(u, v)$ be a regular parameterization of a smooth surface in Euclidean 3-space, and defined by

$$
X(u, v): U \subseteq \mathbb{R}^{2} \rightarrow \mathbb{R}^{3}
$$

The variables $(u, v)$ are called the (curvilinear) coordinates on the surface, the two families of ucurves $(v=$ const), and v-curves $(u=$ const), are called the parametric curves (or coordinate curves). Their directions are defined by the tangents vectors $X_{u}$ and $X_{v}$ respectively. The surface $X(u, v)$ is regular if the condition $X_{u} \times X_{v} \neq 0$ is satisfied for all points, that means the vectors $X_{u}$ and $X_{v}$ do not vanish and have different directions. Consequently, the surface normal is defined at every point on the regular surface as a unit vector on the tangent plane and given by

$$
N(u, v)=\frac{X_{u} \times X_{v}}{\left|X_{u} \times X_{v}\right|}
$$

The first and second fundamental form of the parameterized regular surface are given by

$$
I=E d s^{2}+2 F d s d v+G d v^{2}, \quad I I=e d s^{2}+2 f d s d v+g d v^{2}
$$

where their coefficients can be calculated respectively as

$$
\begin{gathered}
E=<X_{s}, X_{s}>, \quad F=<X_{s}, X_{v}>, \quad G=<X_{v}, X_{v}> \\
e=<N, X_{s s}>, \quad f=<N, X_{v s}>, \quad g=<N, X_{v v}>
\end{gathered}
$$

The fundamental quantities I and II are important tools to describe the intrinsic and extrinsic geometry of surface. In particular, type of the parametric curves and their characteristics properties are described by the coefficients of the fundamental quantities I and II. For example, the coordinate curves are orthogonal if $F=0$, conjugate if $f=0$, and lines of curvature if satisfy both conditions.

Theorem 2.1. [14] A necessary and sufficient condition for the coordinate curves of a parametrization to be lines of curvature in a neighborhood of a nonumbilical point is that $F=f=0$.

The ruled surfaces are defined by moving a straight line on a given curve and parameterized by

$$
X(s, v)=\gamma(s)+v D(s), \quad 0 \leq s \leq \ell, \quad v \in \mathbb{R}
$$

A unit regular curve $\gamma(s)$ is called a base curve (or directrix), and the line passing through $\gamma(s)$ that is parallel to $D(s)$ is called the ruling (or generator). $D(s)$ is a unit director vector field that gives the direction of the ruling. Different ruled surfaces can be constructed based on different $\gamma(s)$ and $D(s)$. By the above discussion, the ruled surface has two families of parametric curves, the family of lines (or rulings), and the family of base curves (or directrices), which can be defined respectively by

$$
X\left(s_{0}, v\right)=\left\{\gamma\left(s_{0}\right)+v D\left(s_{0}\right), 0 \leq s_{0} \leq \ell\right\} \quad \text { and } \quad X\left(s, v_{0}\right)=\left\{\gamma(s)+v_{0} D(s), v_{0} \in \mathbb{R}\right\}
$$

In this paper, we call $\gamma(s)$ the first directrix curve, where $X(s, 0)=\gamma(s)$. The unit normal vector field (surface normal, for shortly) is defined by

$$
N(s, v)=\frac{X_{s} \times X_{v}}{\left|X_{s} \times X_{v}\right|}=\frac{\left[\alpha^{\prime}(s)+v D^{\prime}(s)\right] \times D(s)}{\left[\alpha^{\prime}(s)+v D^{\prime}(s)\right] \times D(s) \mid}
$$

A point on a ruled surface that satisfies $X_{s} \times X_{v}=0$ is called a singular point. A ruled surface may have singular points, which are located (if exist) on the striction curve that parameterized by [12]

$$
\beta(s)=\gamma(s)-\frac{\left\langle\gamma^{\prime}(s), D^{\prime}(s)\right\rangle}{\left\langle D^{\prime}(s), D^{\prime}(s)\right\rangle} D(s), \quad D^{\prime}(s) \neq 0
$$

A ruled surface is said to be cylindrical if $\mathrm{D}(\mathrm{s})$ is constant, i.e. $D^{\prime}(s)=0$. Otherwise, it is said to be non-cylindrical. The Gaussian curvature is non-positive for a ruled surface, it vanishes identically for special classes called the developable surfaces. Equivalently, a ruled surface (17) is developable if and only if $[12]$

$$
\operatorname{det}\left\langle\gamma^{\prime}(s), D(s), D^{\prime}(s)\right\rangle=0
$$


Remark 2.2. For a developable surface, the singular points can be classified using (20) into three cases based on the type of developable surface.

i. Cylindrical surface (all rulings are parallel i.e. $D^{\prime}(s)=0$ ), has no singular points.

ii. Conical surface (all rulings meet at a vertex ), has one singular point at its vertex.

iii. Tangent surface has singularities along the curve which is called the cuspidal edge.

A curve is called a twisted curve if has nonzero curvature and torsion. Through this paper, we assume that the first directrix curve $\gamma(s)$ is a unit speed twisted curve. $D(s)$ is a unit director vector field lies in the space formed by moving frame $\{t, n, b\}$ of $\gamma(s)$ and can be written using (7) as

$$
D(s)=\cos \theta(s) t(s)+\sin \theta(s) g(s), \text { where } g(s)=\cos \phi(s) n(s)+\sin \phi(s) b(s)
$$

Therefore, $D(s)$ can be decomposed as following [15]

$$
D(s)=\cos \theta(s) t(s)+\sin \theta(s)(\cos \phi(s) n(s)+\sin \phi(s) b(s))
$$

Definition 2.3. A surface defined by:

$$
X(s, v)=\gamma(s)+v D(s), \quad 0 \leq s \leq L, \quad \text { and } \quad v \in \mathbb{R}
$$

is ruled surface where $D(s)=\cos \theta(s) t(s)+\sin \theta(s)(\cos \phi(s) n(s)+\sin \phi(s) b(s))$.

The singular points on the constructed ruled surface are avoided, therefore the vectors $\gamma^{\prime}(s)$ and $D(s)$ must be not collinear. It requires that $\sin \theta(s) \neq 0$ which can be used as a regularity condition. By using (8) the surface normal along the first directrix curve is given by

$$
N(s, 0)=-\sin \phi(s) n(s)+\cos \phi(s) b(s)
$$

Lemma 2.4. [15] A ruled surfaces family (24) is developable if and only if the following condition is satisfied,

$$
\sin \theta(s)\left(\frac{d \phi}{d s}+\tau(s)\right)-\kappa(s) \sin \phi(s) \cos \theta(s)=0
$$

The main result of this paper is the following main theorem which is proved in the next section.

Theorem 2.5. Let $X(s, v)=\gamma(s)+v D(s), 0 \leq s \leq L, v \in \mathbb{R}$ be a ruled surface, where $\gamma(s)$ is a unit speed twisted curve, and $D(s)$ is a unit director vector defined by (23), then the developable surface with geodesic coordinates is a generalized cylinder. And the developable surface with lines of curvatures coordinates is a tangent surface.

\section{Developable Surface with Geodesic and Line of Curvature Coordinates}

This section is the main part of this paper, it consists of two subsections which are devoted to parameterizing the developable surface with geodesics and lines of curvature coordinate respectively. As well known, the parametric curves of the developable surface are base curves (directrices) and lines (rulings). Any part of a straight line on a developable surface is geodesic and line of curvature, therefore, this section is devoted to giving the necessary and sufficient condition for the directrices to be geodesics or lines of curvature. We show that the developable surface with geodesic coordinates is a generalized cylinder, and the developable surface with line of curvature coordinates is a tangent surface. 


\subsection{Developable Surface with Geodesics Coordinates}

This subsection is devoted to construct a ruled developable surface parameterized by (24) with geodesic coordinates. In particular, to design a developable surface with geodesic directrices curves. For this purpose, three conditions must be satisfied through three theorems, the first one is a geodesic condition (10) that insures the first directrix curve is a geodesic. The second one is the developability condition (26). Finally, the third condition is that makes the other directrices are geodesics. In the following, we start with the first theorem which gives the condition that makes the first directrix curve is a geodesic.

Theorem 3.1. Let $\mathrm{X}$ be a ruled surface parameterized by $X(s, v)=\gamma(s)+v D(s), 0 \leq s \leq L, v \in \mathbb{R}$, where $\gamma(s)$ is the first directrx curve. $D(s)$ is a unit director vector defined by $(23)$, then $\gamma(s)$ is a geodesic if and only if $D(s)$ is a rectifying vector.

Proof. From (25), it is clear that $N= \pm n$, if and only if $\cos \phi(s)=0$, by (10) this is equivalent to $\gamma(s)$ is a geodesic if and only if $D(s)$ is a rectifying vector.

Definition 3.2. A ruled surface defined by:

$$
X(s, v)=\gamma(s)+v D(s), \quad 0 \leq s \leq L, \quad \text { and } \quad v \in \mathbb{R}
$$

possesses the first directrix curve as geodesic where $D(s)=\cos \theta(s) t(s)+\sin \theta(s) b(s)$.

The following theorem gives the second condition that makes the ruled surface with geodesic first directrix (27) is a developable

Theorem 3.3. A ruled surface that has a geodesic first directrix (27) is developable if and only if $\tau(s) \sin \theta(s)-\kappa(s) \cos \theta(s)=0$.

Proof. Since $\cos \phi(s)=0$ for a ruled surface whose the first directrix is a geodesic (27), then the developability condition (26) can be abbreviated as

$$
\tau(s) \sin \theta(s)-\kappa(s) \cos \theta(s)=0
$$

Definition 3.4. A ruled surface defined by:

$$
X(s, v)=\gamma(s)+v[\cos \theta(s) t(s)+\sin \theta(s) b(s)], \quad 0 \leq s \leq L, \quad \text { and } \quad v \in \mathbb{R}
$$

is a developable surface with geodesic first directrix where $\tau(s) \sin \theta(s)-\kappa(s) \cos \theta(s)=0$.

The developability condition (28) for a ruled surface whose first directrix curve is a geodesic has geometric restriction should be imposed on the unit director vector as given in the following proposition

Proposition 3.5. [16] Suppose that $D(s)=\cos \theta(s) t(s)+\sin \theta(s) b(s)$ is a rectifying vector field defined along a unit speed twisted curve $\gamma(s)$, then $D(s)$ is a unit Darboux vector field if and only if $\kappa \cos \theta-\tau \sin \theta=0$

Proof. Let $D(s)=\cos \theta(s) t(s)+\sin \theta(s) b(s)$ be a unit Darboux vector. From (3),

$$
\cos \theta=\frac{\tau}{\sqrt{\kappa^{2}+\tau^{2}}}, \quad \sin \theta(s)=\frac{\kappa}{\sqrt{\kappa^{2}+\tau^{2}}}, \quad \text { and } \quad \cot \theta=\frac{\tau}{\kappa}
$$

This implies that $\kappa \cos \theta-\tau \sin \theta=0$, and vice versa.

Definition 3.6. A ruled surface defined by:

$$
X(s, v)=\gamma(s)+v D(s), \quad 0 \leq s \leq L, \quad \text { and } \quad v \in \mathbb{R}
$$

is developable with geodesic first directrix where $D(s)=\frac{\tau(s)}{\sqrt{\kappa^{2}+\tau^{2}}} t(s)+\frac{\kappa(s)}{\sqrt{\kappa^{2}+\tau^{2}}} b(s)$. 
The developable surface whose first directrix curve is a geodesic has an equivalent two parameterization (29) and (30). The parameterization (30) has a nice geometric expression, where the constraints are viewed on the director vector, whereas the calculations based on differentiation become easier with parameterization (29). This can be shown as follows, where the first and second derivatives of the developable surface with geodesic first directrix (29) are listed in the following equations

$$
\left\{\begin{array}{l}
X_{s}=\left(1-v \sin \theta \theta^{\prime}(s)\right) t+v \cos \theta \theta^{\prime}(s) b \\
X_{s s}=-v\left(\sin \theta \theta^{\prime \prime}+\cos \theta \theta^{\prime 2}\right) t+\left[\kappa-v \theta^{\prime}(\kappa \sin \theta+\tau \cos \theta)\right] n+v\left(\cos \theta \theta^{\prime \prime}-\sin \theta \theta^{\prime 2}\right) b \\
X_{s v}=-\sin \theta \theta^{\prime}(s) t(s)+v \cos \theta \theta^{\prime}(s) b(s) \\
X_{v}=\cos \theta(s) t(s)+\sin \theta(s) b(s) \\
X_{v v}=0
\end{array}\right.
$$

The inner and cross products of the tangents vectors $X_{s}$ and $X_{v}$ are given by

$$
\left\{\begin{array}{l}
\left\langle X_{s}, X_{v}\right\rangle=\cos \theta(s) \\
X_{s} \times X_{v}=\left(-\sin \theta(s)+v \theta^{\prime}(s)\right) n(s)
\end{array}\right.
$$

By using the regularity condition $\sin \theta(s) \neq 0$, and from (32) the unit normal of developable surface with geodesic first directrix (29) is defined everywhere and given by the following

$$
N(s, v)=\frac{X_{s} \times X_{v}}{\left|X_{s} \times X_{v}\right|}=n
$$

Until now, we get a parameterization (30) that is characterized by two properties, the first directrix curve is geodesic and the ruled surface is developable. These are satisfied under two constraints, the director vector must be rectifying, and Darboux vector receptively. The following third theorem gives the third condition that makes the directrices are geodesics as one of the main results of this paper.

Theorem 3.7. Let $X(s, v)$ be a developable surface parameterized by (29). Then, the directrices curves are geodesics if and only if $\theta$ is constant.

Proof. The directrices curves on a developable surface (29) are geodesics if and only if its acceleration vector $X_{s s}$ is normal to the surface according to (11), then we have

$$
N(s, v) \times X_{s s}=0
$$

From (31) and (33), it follows that

$$
-v\left(\sin \theta \theta^{\prime \prime}+\cos \theta \theta^{\prime 2}\right) b+v\left(\cos \theta \theta^{\prime \prime}-\sin \theta \theta^{\prime 2}\right) t=0
$$

The above condition is satisfied when $v=0$, i.e., the first directrix curve is geodesic. Or the coefficients of tangent and binormal vectors must vanish simultaneously as the following

$$
\left\{\begin{array}{l}
\sin \theta \theta^{\prime \prime}+\cos \theta \theta^{\prime 2}=0 \\
\cos \theta \theta^{\prime \prime}-\sin \theta \theta^{\prime 2}=0
\end{array}\right.
$$

Using the elimination method, we obtain their common solution $\theta^{\prime}=0$, which implies that $\theta$ is a constant and vice versa.

Definition 3.8. A ruled surface defined by:

$$
X(s, v)=\gamma(s)+v[\cos \theta(s) t(s)+\sin \theta(s) b(s)], \quad 0 \leq s \leq L, \quad \text { and } \quad v \in \mathbb{R}
$$

is developable with geodesic directrices where $\tau(s) \sin \theta(s)-\kappa(s) \cos \theta(s)=0$ and $\theta^{\prime}(s)=0$. 
As we know from poposition (3.5), the second condition $\tau(s) \sin \theta(s)-\kappa(s) \cos \theta(s)=0$ means that the unit director vector is a unit Darboux vector. At the same time, the third condition, $\theta^{\prime}(s)=0$, implies that a unit Darboux vector is constant.

Definition 3.9. A ruled surface defined by:

$$
X(s, v)=\gamma(s)+v D(s), \quad 0 \leq s \leq L, \quad \text { and } \quad v \in \mathbb{R}
$$

is developable with geodesic directrices where $D(s)=\frac{\tau(s)}{\sqrt{\kappa^{2}+\tau^{2}}} t(s)+\frac{\kappa(s)}{\sqrt{\kappa^{2}+\tau^{2}}} b(s)$ and $D^{\prime}(s)=0$.

There is an important hidden property in an equivalent parameterizations (36) and (37) for a developable surface with geodesic directrices, and can be extracted by the following proposition

Proposition 3.10. For a developable surface with geodesic directrices and parametrized by (36) or (37), the first directrix is a helix.

Proof. For parameterization (36), we have the second condition

$$
\tau(s) \sin \theta(s)-\kappa(s) \cos \theta(s)=0, \quad \text { which gives } \cot \theta=\frac{\tau}{\kappa}
$$

At the same time, we have the third condition $\theta^{\prime}(s)=0$, which implies that $\frac{\tau}{\kappa}=c$, then the first directrix is a helix by definition. For parameterization (37), the unit Darboux vector is a constant, which means that the first directrix is a helix as discussed in (4), which confirms that every helix described by its constant unit Darboux vector.

The first directrix curve and the director vector are responsible to build the ruled developable surface, therefore the following theorem gives the conditions that can be applied on the first directrix curve and the director vector at the same time to generate a developable parameterization with geodesic directrices.

Theorem 3.11. Let $X(s, v)=\gamma(s)+v D(s), 0 \leq s \leq L, v \in \mathbb{R}$ be a ruled surface, where $\gamma(s)$ is a unit speed twisted curve, $D(s)$ is a unit director vector defined by (23). Then every ruling is geodesic and the directrices curves are geodesics if and only if $\gamma(s)$ is a helix and $D(s)$ is a unit Darboux vector.

Definition 3.12. A ruled surface defined by:

$$
X(s, v)=\gamma(s)+v D(s), \quad 0 \leq s \leq L, \quad \text { and } \quad v \in \mathbb{R}
$$

is developable with geodesic directrices where $D(s)=\frac{\tau(s)}{\sqrt{\kappa^{2}+\tau^{2}}} t(s)+\frac{\kappa(s)}{\sqrt{\kappa^{2}+\tau^{2}}} b(s)$ and $\gamma(s)$ is a helix.

The developable surface with geodesic directrices is a generalized cylinder, where the director vector is constant, i.e., $D^{\prime}(s)=0(37)$. Therefore, the generalized cylinder is the only developable surface that can be coordinated by geodesics parametric curves with additional assumptions. This result is given in the following theorem

Theorem 3.13. Among all developable surfaces parameterized by (24), the generalized cylinder can be equipped with geodesic coordinates if and only if the directrix curve is a helix and the director vector is a unit Darboux vector.

For a developable surface with geodesic coordinates (38), the directrices geodesic curves have the same curvature and torsion, and differ only by the rigid motion modeled by a constant unit Darboux vector with fixed direction and fixed angular velocity, this result formulated as following

Proposition 3.14. Let $X(s, v)=\gamma(s)+v D(s), 0 \leq s \leq L, v \in \mathbb{R}$, be a ruled surface, $D(s)$ is a unit director vector defined by (23), if the directrices are geodesics, then they are congruent.

Corollary 3.15. The geodesic parametric curves of the developable surface (38) are lines and helices.

Corollary 3.16. There is no a skew ruled surface parameterized by (24) whose parametric curves are geodesics. 


\subsection{Developable Surface with Line of Curvature Coordinates}

This subsection investigates how to construct a ruled developable surface parametrized by (24) with lines of curvature coordinates. In particular, to design a developable surface with lines of curvature directrices curves. For this aim, two conditions are required, the first one insures that the first directrix curve is a line of curvature. The second one is a developability condition (26). After that, we show that the other directrices of a ruled developable surface are lines of curvature without further assumptions. The following definition combines the line of curvature condition (12) with parametrization (24) to obtain a ruled surface whose the first directrix curve is a line of curvature.

Definition 3.17. A ruled surface defined by:

$$
X(s, v)=\gamma(s)+v[\cos \theta(s) t(s)+\sin \theta(s)(\cos \phi(s) n(s)+\sin \phi(s) b(s))], \quad 0 \leq s \leq L, \quad \text { and } \quad v \in \mathbb{R}
$$

has line of curvature first directrix where $\tau+\frac{d \phi}{d s}=0$.

Remark 3.18. If $\cos \phi(s)=0$ or $\sin \phi(s)=0$, then $\phi(s)$ has constant value and $\frac{d \phi}{d s}=0$, this implies that $\tau=0$, it is contradiction with the assumption that $\gamma(s)$ is a twisted curve. In general, $\cos \phi(s)=$ const. or $\sin \phi(s)=$ const., lead to the same contradiction. Therefore, through this section we suppose that neither $\cos \phi(s)=$ const. nor $\sin \phi(s)=$ const.

Theorem 3.19. A ruled surface with line of curvature first directrix (39) is developable if and only if $\cos \theta(s)=0$.

Proof. A ruled surface whose first directrix is the line of curvature (39) satisfies $\tau+\frac{d \phi}{d s}=0$. After substitution, the developabity condition (26) becomes

$$
\kappa(s) \sin \phi(s) \cos \theta(s)=0
$$

Since $\gamma(s)$ is a twisted curve, then $\sin \phi(s) \neq 0$ and $\kappa(s) \neq 0$. Therefore, a ruled surface with line of curvature first directrix (39) is developable if and only if $\cos \theta(s)=0$.

Definition 3.20. A ruled surface defined by:

$$
X(s, v)=\gamma(s)+v[\cos \phi(s) n(s)+\sin \phi(s) b(s)], \quad 0 \leq s \leq L, \quad \text { and } \quad v \in \mathbb{R}
$$

with line of curvature first directrix is developable where $\tau+\frac{d \phi}{d s}=0$.

The first and second derivatives of the developable surface parametrization whose the first directrix is line of curvature (41) are listed in the following equations

$$
\left\{\begin{array}{l}
X_{s}=(1-v \kappa \cos \phi(s)) t(s) \\
X_{s s}=-v\left(\frac{d \kappa}{d s} \cos \phi(s)-\kappa \sin \phi(s) \frac{d \phi(s)}{d s}\right) t(s)+\kappa(1-v \kappa \cos \phi(s)) n(s) \\
X_{s v}=-\kappa \cos \phi(s) t(s) \\
X_{v}=\cos \phi(s) n(s)+\sin \phi(s) b(s) \\
X_{v v}=0
\end{array}\right.
$$

The inner and cross products of the tangents vectors $X_{s}$ and $X_{v}$ are given by

$$
\left\{\begin{array}{l}
\left\langle X_{s}, X_{v}\right\rangle=0 \\
X_{s} \times X_{v}=(1-v \kappa \cos \phi(s))[-\sin \phi(s) t(s)+\cos \phi(s) b(s)]
\end{array}\right.
$$

By using the regularity condition $1-v \kappa \cos \phi(s) \neq 0$, and from (43) the unit normal of developable surface with line of curvature first directrix (41) is defined everywhere and given by the following

$$
N(s, v)=\frac{X_{s} \times X_{v}}{\left|X_{s} \times X_{v}\right|}=-\sin \phi(s) n(s)+\cos \phi(s) b(s)
$$


Theorem 3.21. Let $\mathrm{X}$ be a developable surface parameterized by (41), then the directrices curves are line of curvature.

Proof. By Theorem (2.1), the directrices curves on a developable surface (41) are line of curvature if and only if $F=f=0$. First, its clearly that $F=\left\langle X_{s}, X_{v}\right\rangle=0$ from equation (43). Second, using equations (42) and (44), we obtain that

$$
f=<N, X_{v s}>=<-\sin \phi(s) n(s)+\cos \phi(s) b(s),-\kappa \cos \phi(s) t(s)>=0
$$

Theorem (3.21) proves that for the developable parametrization (41), the condition $\tau+\frac{d \phi}{d s}=0$ for the first directrix to be a line of curvature is sufficient to make other directrices are lines of curvature.

Theorem 3.22. Let $X(s, v)=\gamma(s)+v D(s), 0 \leq s \leq L, v \in \mathbb{R}$ be a developable ruled surface, where $\gamma(s)$ is a unit speed twisted curve, and $D(s)=\cos \phi(s) n(s)+\sin \phi(s) b(s)$ is a unit director vector. Then every ruling is line of curvature and the directrices curves are lines of curvature if and only if $\tau+\frac{d \phi}{d s}=0$.

Previously, the condition $\tau+\frac{d \phi}{d s}=0$ with this form has been used to simplify the calculations. For the surface construction, it is useful to write the condition in terms of $\phi(s)$ explicitly as $\phi(s)=$ $\phi_{0}-\int_{s_{0}}^{s} \tau d s$. If we choose $s_{0}=0$, hence $\phi_{0}=\phi(0)$, then the condition becomes $\phi(s)=\phi(0)-\int_{0}^{s} \tau d s$.

Definition 3.23. A ruled surface defined by:

$$
X(s, v)=\gamma(s)+v[\cos \phi(s) n(s)+\sin \phi(s) b(s)], \quad 0 \leq s \leq L, \quad \text { and } \quad v \in \mathbb{R}
$$

is developable surface with line of curvature coordinates where $\phi(s)=\phi(0)-\int_{0}^{s} \tau d s$.

As we have seen, the developable surface with geodesic coordinates is a generalized cylinder. The type of developable surface with line of curvature coordinates (46) is determined in the following.

Theorem 3.24. A developable surface with line of curvature coordinates (46) is non cylindrical.

Proof. Assume by contradiction that a developable surface with line of curvature coordinates (46) is a cylinder, then $D^{\prime}(s)=0$. Since $D(s)=\cos \phi(s) n(s)+\sin \phi(s) b(s)$, then $D^{\prime}(s)=-\kappa \cos \phi(s) t(s)=0$ implies that $\kappa=0$ or $\cos \phi=0$, which contradicts to the assumption that $\gamma(s)$ is a twisted curve. Hence $D^{\prime}(s) \neq 0$, and a developable surface with line of curvature coordinates (41) is non cylindrical.

Non-cylindrical developable surface is a tangent or a generalized cone surfaces. The singular points or the striction curve $\beta(s)$ (20) can be used to distinguish between them as given in the following.

Definition 3.25. A non-cylindrical developable surface is

i. A generalized cone if and only if the striction curve $\beta(s)(20)$ is a fixed point, i.e., $\beta^{\prime}(s)=0$.

ii. A tangent surface if and only if, there exists striction curve $\beta(s)(20)$ such that $\beta^{\prime}(s) \neq 0$.

Theorem 3.26. For a developable surface with line of curvature coordinates (46). The striction curve and its first derivative are given respectively by

$$
\left\{\begin{array}{l}
\beta(s)=\gamma(s)+\frac{1}{\kappa \cos \phi} D(s) \\
\beta^{\prime}(s)=\frac{\kappa \sin \phi \phi^{\prime}(s)-\kappa^{\prime} \cos \phi}{\kappa^{2} \cos ^{2} \phi} D(s)
\end{array}\right.
$$


Proof. The striction curve for non-cylindrical developable surface is defined by

$$
\beta(s)=\gamma(s)-\frac{\left\langle\gamma^{\prime}(s), D^{\prime}(s)\right\rangle}{\left\langle D^{\prime}(s), D^{\prime}(s)\right\rangle} D(s), D^{\prime}(s) \neq 0
$$

For a developable surface with line of curvature coordinates $(46),\left\langle\gamma^{\prime}(s), D^{\prime}(s)\right\rangle=-\kappa \cos \phi$ and $\left\langle D^{\prime}(s), D^{\prime}(s)\right\rangle=\kappa^{2} \cos ^{2} \phi$. Then, the striction curve (20) is given by

$$
\beta(s)=\gamma(s)+\frac{1}{\kappa \cos \phi} D(s)
$$

The first derivative of the striction curve is

$$
\begin{aligned}
\beta^{\prime}(s) & =t(s)+\frac{\kappa \cos \phi(-\kappa \cos \phi t(s))-\left(\kappa^{\prime} \cos \phi-\kappa \sin \phi \phi^{\prime}(s)\right) D(s)}{\kappa^{2} \cos ^{2} \phi} \\
& =\frac{\kappa \sin \phi \phi^{\prime}(s)-\kappa^{\prime} \cos \phi}{\kappa^{2} \cos ^{2} \phi} D(s)
\end{aligned}
$$

Theorem 3.27. A non cylindrical developable surface with line of curvature coordinates (46) is a tangent surface.

Proof. Suppose that a non cylindrical developable surface with lines of curvature coordinates (46) is a generalized cone, then $\beta^{\prime}(s)=0$. By using (47), the following equation is satisfied

$$
\kappa \sin \phi \phi^{\prime}(s)-\kappa^{\prime} \cos \phi=0, \text { which gives } \frac{\kappa^{\prime}}{\kappa}=\frac{\sin \phi}{\cos \phi} \phi^{\prime}(s)
$$

By taking the integral of both sides and some calculations, we get

$$
\kappa \cos \phi=\kappa_{0} \cos \phi_{0}
$$

Since $\kappa_{0}$ and $\cos \phi_{0}$ are constants, then $\kappa$ and $\cos \phi$ are both also constants, this yields a contradiction with a twisted curve. Therefore $\beta^{\prime}(s) \neq 0$, and a non cylindrical developable surface with line of curvature coordinates (46) must be a tangent surface, where $\beta^{\prime}(s)$ is parallel with director $D(s)$.

Li et al. [11] derived a similar condition with (48) in order to make the developable surface through a given line of curvature is cone or tangent surface. Finally, the following theorem is more identified.

Theorem 3.28. Among all developable surfaces parametrized by (24), the tangent surface can be equipped with lines of curvature coordinates if and only if the unit director vector is $D(s)=$ $\cos \phi(s) n(s)+\sin \phi(s) b(s)$ where $\phi(s)=\phi(0)-\int_{0}^{s} \tau d s$.

\section{Examples}

In this section we give an example of a developable surface whose coordinates is geodesic (generalized cylinder) or line of curvature (tangent surface) and draw their pictures by using Mathematica.

Example 4.1. Let $\gamma(s)=\left(\frac{\sqrt{3}}{2} \sin (s), \frac{s}{2}, \frac{\sqrt{3}}{2} \cos (s)\right)$ be a unit speed circular helix curve, the unit tangent, the unit normal and the unit binormal are $t=\left(\frac{\sqrt{3}}{2} \cos (s), \frac{1}{2},-\frac{\sqrt{3}}{2} \sin (s)\right), n=(-\sin (s), 0,-\cos (s))$ and $b=\left(-\frac{1}{2} \cos (s), \frac{\sqrt{3}}{2}, \frac{1}{2} \sin (s)\right)$. The curvature and torsion of $\alpha(s)$ are $\kappa=\frac{\sqrt{3}}{2}$ and $\tau=\frac{1}{2}$.

(a) According to theorem (3.11) and definition (38), the developable geodesic coordinates is given by

$$
X(s, v)=\gamma(s)+v D(s), \quad 0 \leq s \leq L, \quad \text { and } \quad v \in \mathbb{R}
$$

where $D(s)=\frac{\tau(s)}{\sqrt{\kappa^{2}+\tau^{2}}} t(s)+\frac{\kappa(s)}{\sqrt{\kappa^{2}+\tau^{2}}} b(s)$ and $\gamma(s)$ is a helix.

By substitution $\frac{\tau}{\sqrt{\kappa^{2}+\tau^{2}}}=\frac{1}{2}$ and $\frac{\kappa}{\sqrt{\kappa^{2}+\tau^{2}}}=\frac{\sqrt{3}}{2}$, and for $0 \leq s \leq 2 \pi, 0 \leq v \leq \pi$, the constructed developable surface is a generalized cylinder with geodesic coordinates as shown in Figure 1(a). 


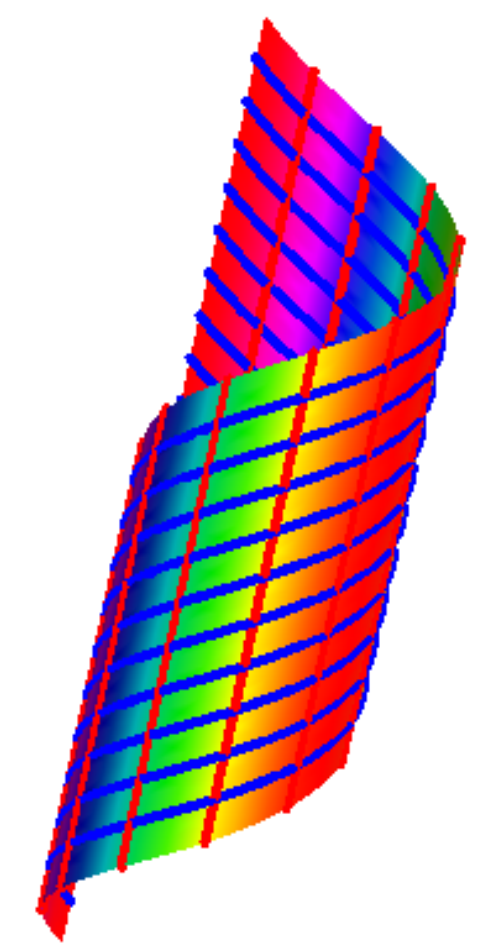

(a) Cylinder with geodesic coordinates.

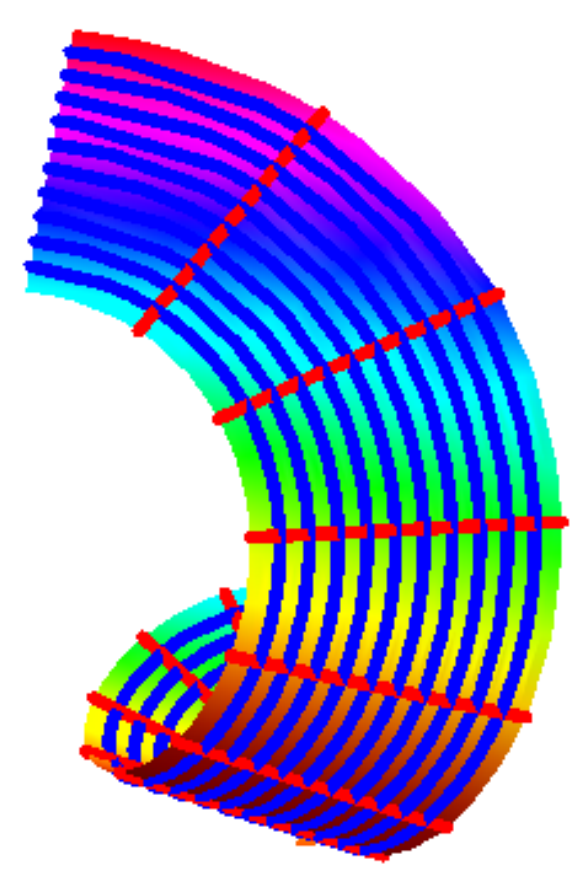

(b) Tangent surface with line of curvature coordinates.

Fig. 1. Developable surface with geodesic or line of curvature coordinates.

(b) By theorem (3.22) and definition (46), the developable line of curvature coordinates is given by

$$
X(s, v)=\gamma(s)+v[\cos \phi(s) n(s)+\sin \phi(s) b(s)], \quad 0 \leq s \leq L, \quad \text { and } \quad v \in \mathbb{R}
$$

where $\phi(s)=\phi(0)-\int_{0}^{s} \tau d s$.

If we choose $\phi(0)=0$, then $\phi(s)=-\frac{1}{2} s$, and for $0 \leq s \leq 2 \pi, 0 \leq v \leq \pi / 2$, the developable constructed surface is a tangent surface with line of curvature coordinates as shown in Figure 1(b).

\section{Conclusion}

In this paper, using a ruled parametrization (24), we constructed a developable surface whose coordinates curves are geodesics or lines of curvature. The main results asserted that the developable surface with geodesic coordinates is a generalized cylinder, and the developable surface with the line of curvature coordinates is a tangent surface. Also, the first directrix curve must be a helix as a first condition to generate a developable surface with geodesic coordinates, but this condition need not to be satisfied to construct a developable surface with the line of curvature coordinates. Furthermore, the developable line of curvature coordinates is orthogonal, but the developable geodesic coordinates is not. Finally, among the three types of developable surfaces, the generalized cylinder and the tangent surface can be equipped with geodesic coordinates and line of curvature coordinates respectively.

\section{Author Contributions}

The author read and approved the last version of the manuscript. 


\section{Conflicts of Interest}

The author declares no conflict of interest.

\section{References}

[1] H. Pottmann, A. Asperl, M. Hofer, A. Kilian, Architectural geometry, Bentley Institute Press, 2007.

[2] Y. Liu, H. Pottmann, J. Wallner, Y. L. Yang, W. Wang, Geometric Modeling with Conical Meshes and Developable Surfaces, In ACM SIGGRAPH Papers (2006) 681-689.

[3] C. Tang, P. Bo, J. Wallner, H. Pottmann, Interactive Design of Developable Surfaces, ACM Transactions on Graphics (TOG) 35(2) (2016) 1-12.

[4] W. K. Schief, On the Integrability of Bertrand Curves and Razzaboni Surfaces, Journal of Geometry and Physics 45(1-2) (2003) 130-150.

[5] N. Gürbüz, The Motion of Timelike Surfaces in Timelike Geodesic Coordinates, International Journal of Mathematical Analysis 4 (2010) 349-356.

[6] Y. Li, C. Chen, The Motion of Surfaces in Geodesic Coordinates and 2+ 1-dimensional Breaking Soliton Equation, Journal of Mathematical Physics 41(4) (2000) 2066-2076.

[7] E. Adiels, M. Ander, C. Williams, Brick Patterns on Shells Using Geodesic Coordinates, In Proceedings of IASS Annual Symposia 23 (2017) 1-10 Hamburg, Germany.

[8] X. Tellier, C. Douthe, L. Hauswirth, O. Baverel, Surfaces with Planar Curvature Lines: Discretization, Generation and Application to the Rationalization of Curved Architectural Envelopes, Automation in Construction 106 (2019) p.102880.

[9] H. Zhao, G. Wang, A New Method for Designing a Developable Surface Utilizing the Surface Pencil through a Given Curve, Progress in Natural Science 18(1) (2008) 105-110.

[10] R. A. Al-Ghefaria, A. B. Rashad, An Approach for Designing a Developable Surface with a Common Geodesic Curve, International Journal of Contemporary Mathematical Sciences 8(18) (2013) 875-891.

[11] C. Y. Li, R. H. Wang, C. G. Zhu, An Approach for Designing a Developable Surface through a Given Line of Curvature, Computer-Aided Design 45(3) (2013) 621-627.

[12] M. D. Carmo, Differential Geometry of Curves and Surfaces, Prentice Hall, New Jersey, 1976.

[13] A. N. Pressley, Elementary Differential Geometry, Springer Science \& Business Media, 2010.

[14] F. Doğan, Y. Yaylı, The Relation between Parameter Curves and Lines of Curvature on Canal Surfaces, Kuwait Journal of Science 44(1) (2017) 29-35.

[15] M. I. Shtogrin, Bending of a Piecewise Developable Surface, Proceedings of the Steklov Institute of Mathematics 275(1) (2011) 133-54.

[16] N. M. Althibany, Classification of Ruled Surfaces Family with Common Characteristic Curve in Euclidean 3-space, Turkish Journal of Science (2021) In Press. 